in 1993 (A A Dementieva).

- Photographic catalogue of fields of 100 radio stars in the Northern hemisphere. Work started in 1994 (N V Narignaya).

\title{
4 Discussion
}

The Chairman (L V Morrison) summarised the following areas of overlapping interest and invited discussion on collaboration, possibly by forming new Working Groups.

- Meridian Circle CCD programmes - V 16- $\sigma \sim 50$ mas

Sloan Digital Sky Survey $-\mathrm{V} \sim 18-\sigma \sim 50$ mas

- Photographic surveys

FON - fourfold coverage of Northern sky

USNO 8-inch twin astrograph red-plate survey

FOKAT - Southern hemisphere - remeasure

AGK2 - Northern hemisphere - remeasure

- POSS/UK Schmidt plate measurement

RGO, APM Cambridge, UK

USNO, Flagstaff, USA

STScI, Baltimore, USA

Minnesota, USA

- $\mathrm{AC}$ reduction

ARI, Gerniany

USNO, USA

Sternberg Inst., Russia

- Linking optical and radio reference frames

Several groups are using faint reference stars in the fields of extragalactic radio sources to link the optical to the primary radio frame.

- Astrolabe projects

International catalogue projects in $\mathrm{N}$ and $\mathrm{S}$ hemispheres.

After some discussion it was decided to refer the coordination of the work on selecting reference stars for linking the optical and radio reference frames to the WG on Star Lists.

Given the early stage of development of most of the Meridian Circle CCD programmes, it was considered premature to form a WG to coordinate their observational programmes.

There was no consensus on forming a WG for the POSS measurement projects. 
It was decided to form a new WG to consider the re-measurement of some of the AC zones. The provisional list of members comprised: Jones (RGO, Chairman), Bucciarelli (STScI), Corbin (USNO), Dahn (USNO), de Vegt (Hamburg), Röser (ARI), Urban (USNO, Consulting), Smart (Torino, Consulting).

The astrolabe group already have an active WG to coordinate their programmes. 